\title{
A Method for the Rapid Synthesis of Highly Functionalized 2-Hydroxy-1-Naphthoates. Syntheses of the Naphthoic Acid Components of Neocarzinostatin Chromophore and N1999A2
}

\author{
Nan Ji, Brad M. Rosen, Andrew G. Myers \\ Department of Chemistry and Chemical Biology, Harvard University \\ Cambridge, Massachusetts 02138
}

\section{Experimental Section:}

General Procedures. All reactions were performed in oven- or flame-dried round bottomed or modified Schlenk flasks fitted with rubber septa under a positive pressure of argon, unless otherwise noted. Air- and moisture-sensitive liquids and solutions were transferred via syringe or stainless steel cannula. Where necessary (so noted), solutions were deoxygenated by alternative freeze (liquid nitrogen)/evacuation/thaw cycles ( $\geq$ three iterations). Organic solutions were concentrated by rotary evaporation (house vacuum, $\sim 25$ Torr) at $23-30{ }^{\circ} \mathrm{C}$. Flash column chromatography was performed as described by Still et al., ${ }^{1}$ employing silica gel (60- $\AA$ pore size,

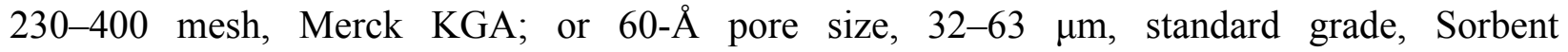
Technologies). Analytical thin-layer chromatography (TLC) was performed using glass plates pre-coated with silica gel $(0.25 \mathrm{~mm}, 60-\AA$ pore size, $230-400$ mesh, Merck KGA) impregnated with a fluorescent indicator $(254 \mathrm{~nm})$. TLC plates were visualized by exposure to ultraviolet light (UV) and/or exposure to ceric ammonium molybdate solution (CAM) or an acidic solution of $p$ anisaldehyde (anisaldehyde) followed by brief heating on a hot plate $\left(\sim 200^{\circ} \mathrm{C}, 10-15 \mathrm{~s}\right)$.

Materials. Commercial reagents and solvents were used as received unless mentioned otherwise. Dichloromethane, ether, tetrahydrofuran, $N, N$-dimethylformamide and toluene were purified by the method of Pangborn et al. ${ }^{2}$ The molarity of solutions of $n$-butyllithium and $t$ butyllithium was determined by titration against a standard solution of 2-butanol in tetrahydrofuran using triphenylmethane as an indicator (average of three determinations). ${ }^{3}$

(1) Still, W. C.; Kahn, M.; Mitra, A. J. Org. Chem. 1978, 43, 2923-2925.

(2) Pangborn, A. B.; Giardello, M. A.; Grubbs, R. H.; Rosen, R. K.; Timmers, F. J. Organometallics 1996, 15, $1518-1520$.

(3) Duhamel, L.; Palquevent, J.-C. J. Org. Chem. 1979, 44, 3404-3405. 
Instrumentation. Proton nuclear magnetic resonance ( ${ }^{1} \mathrm{H}$ NMR) spectra and carbon nuclear magnetic resonance $\left({ }^{13} \mathrm{C}\right.$ NMR) were recorded with Varian Unity/Inova 600 (600 MHz), Varian Unity/ Inova 500 (500 MHz/125 MHz), or Varian Mercury 400 (400 MHz/100 MHz) NMR spectrometers. Chemical shifts for protons are reported in parts per million scale ( $\delta$ scale) downfield from tetramethylsilane and are referenced to residual protium in the NMR solvents $\left(\mathrm{CHCl}_{3}: \delta\right.$ 7.26). Chemical shifts for carbon are reported in parts per million ( $\delta$ scale) downfield from tetramethylsilane and are referenced to the carbon resonances of the solvent $\left(\mathrm{CDCl}_{3}: \delta\right.$ 77.0). Data are represented as follows: chemical shift, multiplicity $(s=$ singlet, $d=$ doublet, $t=$ triplet, $\mathrm{q}=$ quartet, $\mathrm{m}=$ multiplet, $\mathrm{br}=$ broad), integration, coupling constant in $\mathrm{Hz}$, and assignment. Infrared (IR) spectra were obtained using a Perkin-Elmer 1600 FT-IR spectrophotometer referenced to a polystyrene standard. High resolution mass spectra were obtained at the Harvard University Mass Spectrometry Facilities.

\section{List of Abbreviations}

$\begin{array}{ll}\mathrm{AcOH} & \text { acetic acid } \\ \mathrm{CF}_{3} \mathrm{CH}_{2} \mathrm{OH} & \text { trifluoroethanol } \\ \mathrm{CH}_{2} \mathrm{Cl}_{2} & \text { dichloromethane } \\ \mathrm{Cl}_{3} \mathrm{CCCl}_{3} & \text { hexachloroethane } \\ \text { DBU } & 1,8 \text {-diazabicyclo[5.4.0]undec-7-ene } \\ \text { DMF } & N, N \text {-dimethylformamide } \\ E & \text { Ger., entgegen } \\ \text { equiv } & \text { equivalent } \\ \text { ESI } & \text { electrospray ionization } \\ \text { FTIR } & \text { Fourier transform infrared spectroscopy } \\ \text { g } & \text { gram } \\ \text { HRMS } & \text { high resolution mass spectrometry } \\ \mathrm{Hz} & \text { hertz } \\ J & \text { coupling constant }\end{array}$




$\begin{array}{ll}\mathrm{LiOH} & \text { lithium hydroxide } \\ \mathrm{M} & \text { Molarity } \\ \mathrm{mg} & \text { milligram } \\ \mathrm{mL} & \text { milliliter } \\ \mu \mathrm{L} & \text { microliter } \\ \mathrm{mmol} & \text { millimole } \\ \mathrm{Mn}(\mathrm{OAc})_{3} & \text { manganese (III) acetate } \\ \text { mol } & \text { mole } \\ \mathrm{NaH} & \text { sodium hydride } \\ \mathrm{NBS} & N \text {-bromosuccinimide } \\ n \text {-BuLi } & \text { normal butyllithium } \\ \mathrm{NMR} & \text { nuclear magnetic resonance } \\ \text { ppm } & \text { parts per million } \\ \text { THF } & \text { tetrahydrofuran } \\ \text { TIPS } & \text { triisopropylsilyl } \\ Z & \text { Ger., zusammen }\end{array}$




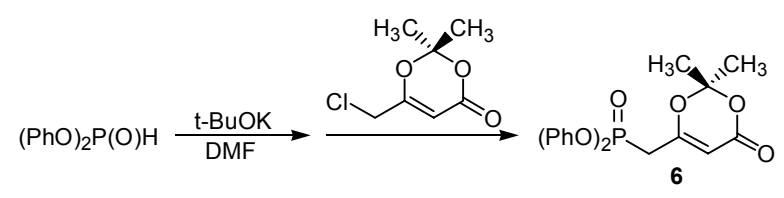

(2,2-Dimethyl-6-oxo-6H-[1,3]dioxin-4-ylmethyl)-phosphonic acid diphenyl ester (6). Diphenyl phosphite (Aldrich, 85\%, containing 15\% phenol, 9.09 g, 38.8 mmol, 3.5 equiv) was added dropwise via syringe over $10 \mathrm{~min}$ to an ice-cooled solution of potassium tert-butoxide (4.36 g, $38.8 \mathrm{mmol}, 3.5$ equiv) in DMF $(50 \mathrm{~mL})$. The resulting solution was allowed to stir at 0 ${ }^{\circ} \mathrm{C}$ for $15 \mathrm{~min}$. A solution of 6-chloromethyl-2,2-dimethyl-4H-1,3-dioxen-4-one (1.96 g, 11.1 mmol, 1 equiv) ${ }^{4}$ in DMF (10.0 mL) was then added via cannula over $2 \mathrm{~min}$. After stirring for $1 \mathrm{~h}$ at $0{ }^{\circ} \mathrm{C}$, the deep purple reaction mixture was allowed to warm to $23{ }^{\circ} \mathrm{C}$. After $9 \mathrm{~h}$ at $23{ }^{\circ} \mathrm{C}$, concentrated aqueous hydrochloric acid solution was added dropwise until the solution turned colorless $(\sim 3 \mathrm{~mL})$. The resulting mixture was partitioned between water $(300 \mathrm{~mL})$ and a 1:1 mixture of ethyl acetate and hexanes $(300 \mathrm{~mL})$. The aqueous layer was separated and extracted with a 1:1 mixture of ethyl acetate and hexanes $(300 \mathrm{~mL})$. The organic extracts were combined and dried over anhydrous sodium sulfate. The dried solution was filtered and the filtrate was concentrated. The residue was purified by flash column chromatography $(50 \%$ ether-hexanes, grading to $67 \%$ ether-hexanes) to afford phosphonate 6 as a white solid, mp $75-77{ }^{\circ} \mathrm{C}(2.52 \mathrm{~g}$, $61 \%)$.

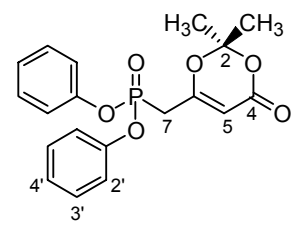

${ }^{1} \mathrm{H}$ NMR (500 MHz, $\mathrm{CDCl}_{3}$ ) $\delta: \quad 7.35$ (t, J=8.0 Hz, 4H, C(3’)-H), 7.23-7.17 (m, 2H, C(4’)Hz; 4H, C(2')-H), 5.50 (d, J=4.0 Hz, 1H, C(5)-H), 3.15 (d, $\mathrm{J}=22.5 \mathrm{~Hz}, 2 \mathrm{H}, \mathrm{C}(7)-\mathbf{H}), 1.69\left(\mathrm{~s}, 6 \mathrm{H}, \mathrm{C}(2)-\left(\mathrm{CH}_{3}\right)_{2}\right)$

${ }^{13} \mathrm{C} \mathrm{NMR}\left(100 \mathrm{MHz}, \mathrm{CDCl}_{3}\right) \delta: \quad 161.7,161.6,160.20,160.18,149.8,149.7,129.9,125.6$, $120.3,120.2,107.4,97.0,96.9,33.0,31.6,24.8$

\footnotetext{
${ }^{4}$ Boeckman, R. K.; Perni, R. B.; Macdonald, J. E.; Thomas, A. J. Organic Syntheses, 1988, 66, 194-202.
} 
HRMS (ESI $\left.{ }^{+}\right)$:

FTIR $\left(\mathrm{cm}^{-1}\right)$ : $m / z$ calcd for $\left(\mathrm{C}_{19} \mathrm{H}_{19} \mathrm{O}_{6} \mathrm{P}+\mathrm{NH}_{4}\right)^{+}$392.1263, found: 392.1255

3500, 3099, 3067, 2995, 2923, 1728, 1634, 1590, 1488, 1376, 1274, 1187, 935 


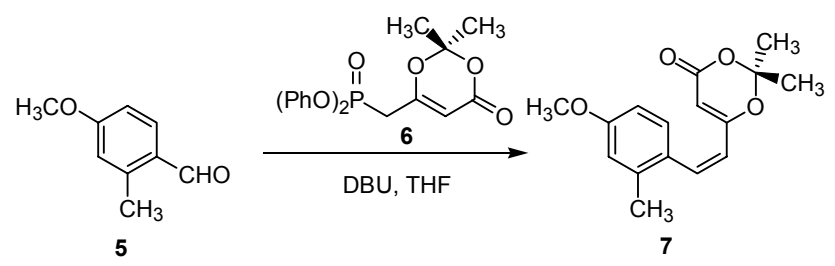

(Z)-Olefin 7. DBU ( $359 \mu \mathrm{L}, 365 \mathrm{mg}, 2.40 \mathrm{mmol}, 2.0$ equiv) was added dropwise via syringe over $2 \mathrm{~min}$ to a stirring solution of phosphonate $6(629 \mathrm{mg}, 1.68 \mathrm{mmol}, 1.4$ equiv) in tetrahydrofuran $(5 \mathrm{~mL})$ at $23{ }^{\circ} \mathrm{C}$. The reaction mixture was allowed to stir at $23^{\circ} \mathrm{C}$ for $20 \mathrm{~min}$. A solution of 4-methoxy-2-methylbenzaldehyde (5) $(181 \mu \mathrm{L}, 200 \mathrm{mg}, 1.20 \mathrm{mmol}, 1$ equiv) in tetrahydrofuran $(5 \mathrm{~mL})$ was added dropwise via cannula. The resulting mixture was allowed to stir at $23{ }^{\circ} \mathrm{C}$ for $18.5 \mathrm{~h}$. Saturated aqueous ammonium chloride solution $(20 \mathrm{~mL})$ was added, and the resulting biphasic mixture was concentrated in vacuo to remove tetrahydrofuran. The aqueous residue was extracted with ether $(3 \times 20 \mathrm{~mL})$. The organic extracts were combined and dried over anhydrous sodium sulfate. The dried solution was filtered and the filtrate was concentrated. The residue was purified by flash column chromatography ( $50 \%$ ether-hexanes) to give (Z)-olefin 7 as a yellow oil (298 mg, $82 \%$ ).

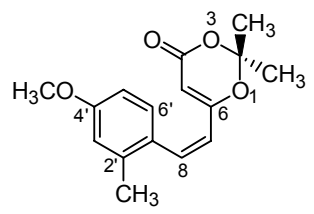

${ }^{1} \mathrm{H}$ NMR (400 MHz, CDCl $)$ ) : 7.11 (d, J=8.4 Hz, 1H, C(6')-H), 6.92 (d, J=12.0 Hz, 1H, C(8)-H), 6.70 (d, J=2.4 Hz, 1H, C(3')-H), 6.66 (dd, J $\mathrm{J}_{1}=2.4$ $\left.\mathrm{Hz}, \mathrm{J}_{2}=8.4 \mathrm{~Hz}, \mathrm{C}\left(5^{\prime}\right)-\mathbf{H}\right), 5.93$ (d, J=12.0 Hz, 1H, C(7)-H), 5.25 (s, 1H, C(5)-H), 3.78 (s, 3H, C(4')-OCH ${ }_{3}$ ), 2.23 (s, 3H, $\left.\mathrm{C}\left(2^{\prime}\right)-\mathrm{CH}_{3}\right), 1.51\left(\mathrm{~s}, 6 \mathrm{H}, \mathrm{C}(2)-\left(\mathrm{CH}_{3}\right)_{2}\right)$

${ }^{13} \mathrm{C} \mathrm{NMR}\left(100 \mathrm{MHz}, \mathrm{CDCl}_{3}\right) \delta: \quad 163.9,161.7,159.6,139.6,137.4,130.2,127.9,121.2$ $114.8,110.5,105.9,95.3,55.1,24.7,20.1$ 
HRMS $\left(\mathrm{ESI}^{+}\right)$:

$\operatorname{FTIR}\left(\mathrm{cm}^{-1}\right)$ : $m / z$ calcd for $\left(\mathrm{C}_{16} \mathrm{H}_{18} \mathrm{O}_{4}+\mathrm{H}\right)^{+} 275.1283$, found 275.1280

3087, 2995, 2944, 2841,1723,1631, 1605, 1564, 1497, $1462,1390,1375,1333,1271,1251,1205,1164,1113$, $1041,1015,903,831$

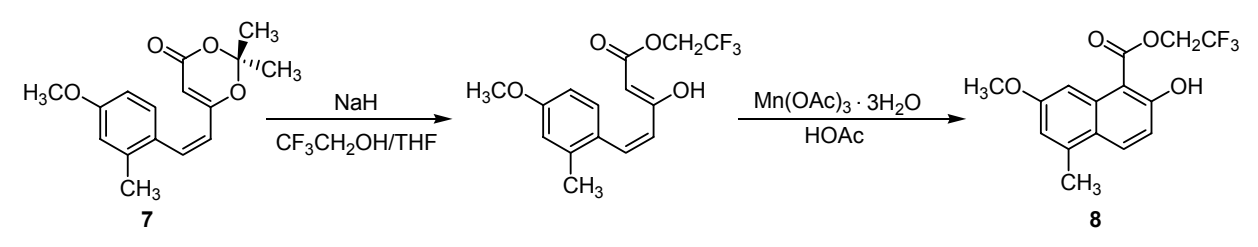

Trifluoroethyl 2-hydroxy-1-naphthoic acid ester 8. Sodium hydride $(60 \%$ dispersion in mineral oil, $51.4 \mathrm{mg}, 2.14 \mathrm{mmol}, 6.0$ equiv) was added to a stirring solution of trifluoroethanol $\left(260 \mu \mathrm{L}, 357 \mathrm{mg}, 3.57 \mathrm{mmol}, 10.0\right.$ equiv) in tetrahydrofuran $(5 \mathrm{~mL})$ at $23{ }^{\circ} \mathrm{C}$. The reaction mixture was allowed to stir at $23{ }^{\circ} \mathrm{C}$ for $20 \mathrm{~min}$. A solution of (Z)-olefin 7 (98.0 mg, $0.357 \mathrm{mmol}$, 1 equiv) in tetrahydrofuran $(5 \mathrm{~mL})$ was added via cannula. After stirring for $30 \mathrm{~min}$ at $23{ }^{\circ} \mathrm{C}$, the resulting mixture was concentrated in vacuo. The residue was taken up in acetic acid $(4 \mathrm{~mL})$. Manganese (III) acetate dihydrate (192 mg, $0.714 \mathrm{mmol}, 2.0$ equiv) was added. After stirring for $20 \mathrm{~h}$ at $23{ }^{\circ} \mathrm{C}$, the reaction mixture was diluted with water $(20 \mathrm{~mL})$. The resulting biphasic mixture was extracted with ethyl acetate $(3 \times 25 \mathrm{~mL})$. The organic extracts were combined and washed with saturated aqueous sodium bicarbonate solution $(2 \times 25 \mathrm{~mL})$. The washed organic solution was dried over anhydrous sodium sulfate. The dried solution was filtered and the filtrate was concentrated. The residue was purified by flash column chromatography $(50 \%$ etherhexanes) to give trifluoroethyl 2-hydroxy-1-naphthoic acid ester 8 as a white solid (104 mg, $93 \%)$.

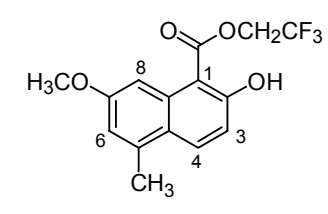


${ }^{1} \mathrm{H}$ NMR (500 MHz, CDCl 3 ) $\delta: \quad 11.78$ (s, 1H, C(2)-OH), 8.06 (d, J=9.5 Hz, 1H, C(4)-H), $7.97(\mathrm{~d}, \mathrm{~J}=2.5 \mathrm{~Hz}, 1 \mathrm{H}, \mathrm{C}(8)-\mathbf{H}), 7.03(\mathrm{~d}, \mathrm{~J}=9.0 \mathrm{~Hz}, 1 \mathrm{H}$, $\mathrm{C}(3)-\mathrm{H}), 6.90$ (d, J=1.0 Hz, 1H, C(6)-H), 4.84 (q, J=8.5 Hz, $\left.2 \mathrm{H}, \mathrm{C}(1)-\mathrm{C}(\mathrm{O}) \mathrm{OCH}_{2} \mathrm{CF}_{3}\right), 3.89$ (s, 3H, C(7)-OCH 3 ), 2.61 (s, $\left.3 \mathrm{H}, \mathrm{C}(5)-\mathrm{CH}_{3}\right)$

${ }^{13} \mathrm{C} \operatorname{NMR}\left(100 \mathrm{MHz}, \mathrm{CDCl}_{3}\right) \delta: \quad 170.9,165.3,159.9,137.1,134.0,133.6,123.2$ (q, J=275 $\mathrm{Hz}), 123.0,117.3,115.8,103.3,103.0,61.4$ (q, J=36.6 Hz), $54.9,19.9$

HRMS (ESI $\left.{ }^{-}\right)$: $m / z$ calcd for $\left(\mathrm{C}_{15} \mathrm{H}_{13} \mathrm{~F}_{3} \mathrm{O}_{4}-\mathrm{H}\right)^{-} 313.0688$, found 313.0694

FTIR $\left(\mathrm{cm}^{-1}\right)$ : 2976, 1672, 1652, 1614, 1415, 1369, 1318, 1276, 1175, $1166,1039,959,846$

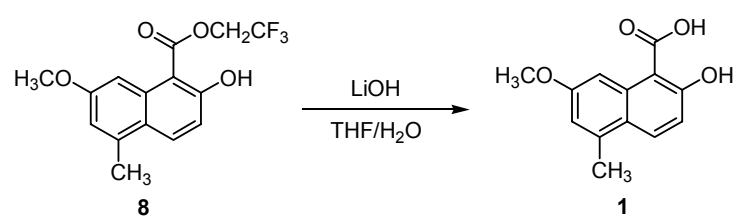

NCS chromophore naphthoic acid (1). Lithium hydroxide monohydrate $(54.5 \mathrm{mg}, 1.30 \mathrm{mmol}$, 20.0 equiv) was added to a stirring solution of trifluoroethyl 2-hydroxy-1-naphthoic acid ester 8 $(20.5 \mathrm{mg}, 0.65 \mathrm{mmol}, 1$ equiv) in tetrahydrofuran $(4.0 \mathrm{~mL})$ and water $(2.0 \mathrm{~mL})$. The resulting biphasic mixture was allowed to stir at $40{ }^{\circ} \mathrm{C}$ for $19 \mathrm{~h}$. Water $(10 \mathrm{~mL})$ was added, the biphasic mixture was cooled to $0{ }^{\circ} \mathrm{C}$, and $1 \mathrm{~N}$ aqueous hydrochloric acid solution was added carefully to an end point of $\mathrm{pH} \sim 1.0(\sim 1.5 \mathrm{~mL})$. The product was extracted with ethyl acetate $(3 \times 15 \mathrm{~mL})$. The organic extracts were combined and washed with brine $(2 \times 10 \mathrm{~mL})$. The washed organic solution was dried over anhydrous sodium sulfate. The dried solution was filtered and the filtrate was concentrated to afford NCS chromophore naphthoic acid (1) as a white solid (15.0 mg, $100 \%)$. 


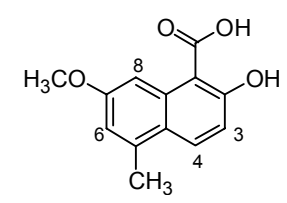

${ }^{1} \mathrm{H}$ NMR (500 MHz, $\left.\mathrm{CDCl}_{3}\right) \delta: \quad 12.02(\mathrm{~s}, \mathrm{br}, 1 \mathrm{H}, \mathrm{C}(2)-\mathrm{OH}), 8.23(\mathrm{~d}, \mathrm{~J}=2.0 \mathrm{~Hz}, 1 \mathrm{H}, \mathrm{C}(8)-\mathbf{H})$, $8.11(\mathrm{~d}, \mathrm{~J}=9.5 \mathrm{~Hz}, 1 \mathrm{H}, \mathrm{C}(4)-\mathbf{H}), 7.06(\mathrm{~d}, \mathrm{~J}=9.5 \mathrm{~Hz}, 1 \mathrm{H}$, C(3)-H), 6.93 (d, J=1.0 Hz, 1H, C(6)-H), 3.95 (s, 3H, C(7)$\left.\mathrm{OCH}_{3}\right), 2.65$ (s, 3H, C(5)-CH $\mathbf{C H}_{3}$

${ }^{13} \mathrm{C} \mathrm{NMR}\left(100 \mathrm{MHz}, \mathrm{CDCl}_{3}\right) \delta: \quad 175.9,165.9,159.9,137.1,134.7,133.9,123.1,116.5$, $116.0,104.2,103.1,55.2,20.0$

HRMS $\left(\mathrm{ESI}^{+}\right)$:

$m / z$ calcd for $\left(\mathrm{C}_{10} \mathrm{H}_{12} \mathrm{O}_{4}+\mathrm{H}\right)^{+} 233.0814$, found 233.0811

FTIR $\left(\mathrm{cm}^{-1}\right)$ : $2930,2829,2705,2627,2548,1634,1620,1589,1449$, $1415,1286,1247,1219,1101,1073,1017,848$ 


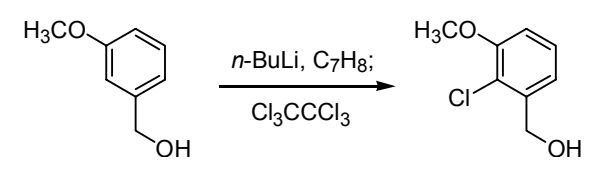

2-Chloro-3-methoxybenzyl alcohol. A solution of $n$-butyllithium in hexanes $(2.5 \mathrm{M}, 19.3 \mathrm{~mL}$, $48.3 \mathrm{mmol}, 3.0$ equiv) was added via syringe over $10 \mathrm{~min}$ to a solution of 3-methoxybenzyl alcohol $\left(2.00 \mathrm{~mL}, 16.1 \mathrm{mmol}, 1\right.$ equiv) in toluene $(70 \mathrm{~mL})$ at $23^{\circ} \mathrm{C}$. After $1 \mathrm{~h}$ at $23^{\circ} \mathrm{C}$, a solution of hexachloroethane (7.62 g, $32.2 \mathrm{mmol}, 2.0$ equiv) in toluene $(20 \mathrm{~mL})$ was added via cannula. Upon completed addition, the reaction mixture was allowed to stir at $23{ }^{\circ} \mathrm{C}$ for $10 \mathrm{~min}$. Saturated aqueous sodium bicarbonate solution $(100 \mathrm{~mL})$ was added, and the resulting biphasic mixture was partitioned between ether $(150 \mathrm{~mL})$ and water $(100 \mathrm{~mL})$. The organic layer was separated and washed with brine $(100 \mathrm{~mL})$. The washed organic solution was dried over sodium sulfate. The dried solution was filtered and filtrate was concentrated. The residue was purified by flash column chromatography (40\% ethyl acetate-hexanes) to afford 2-chloro-3-methoxybenzyl alcohol as a white solid, mp $104-106{ }^{\circ} \mathrm{C}(1.83 \mathrm{~g}, 66 \%)$.

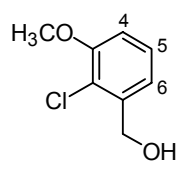

${ }^{1} \mathrm{H}$ NMR (400 MHz, $\left.\mathrm{CDCl}_{3}\right) \delta: \quad 7.26$ (dd, J $\left.=8.4 \mathrm{~Hz}, \mathrm{~J}_{2}=7.6 \mathrm{~Hz}, 1 \mathrm{H}, \mathrm{C}(5)-\mathbf{H}\right), 7.10$ (dd, $\left.\mathrm{J}_{1}=7.6 \mathrm{~Hz}, \mathrm{~J}_{2}=0.8 \mathrm{~Hz}, 1 \mathrm{H}, \mathrm{C}(6)-\mathbf{H}\right), 6.90(\mathrm{~d}, \mathrm{br}, \mathrm{J}=8.4 \mathrm{~Hz}$, $1 \mathrm{H}, \mathrm{C}(4)-\mathbf{H}), 4.79$ (d, J=6.4 Hz, 2H, C(1)-CH $\left.\mathbf{H}_{2} \mathrm{OH}\right), 3.91(\mathrm{~s}$, $\left.3 \mathrm{H}, \mathrm{C}(3)-\mathrm{OCH}_{3}\right), 2.02\left(\mathrm{t}, \mathrm{J}=6.4 \mathrm{~Hz}, 1 \mathrm{H}, \mathrm{C}(1)-\mathrm{CH}_{2} \mathrm{OH}\right)$

${ }^{13} \mathrm{C} \mathrm{NMR}\left(100 \mathrm{MHz}, \mathrm{CDCl}_{3}\right) \delta: \quad 155.0,139.7,127.4,120.8,120.4,111.1,63.0,56.2$

$\operatorname{HRMS}\left(\mathrm{EI}^{+}\right): \quad \quad m / z$ calcd for $\left(\mathrm{C}_{8} \mathrm{H}_{9} \mathrm{ClO}_{2}\right)^{+} 172.0291$, found 172.0283 
$\operatorname{FTIR}\left(\mathrm{cm}^{-1}\right)$ :

$3281,3188,2976,2941,2842,1597,1578,1478,1361$, $1298,1284,1049,766$

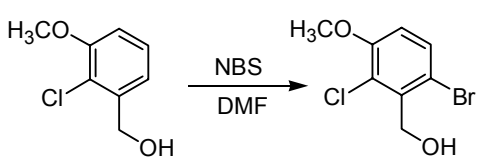

6-Bromo-2-chloro-3-methoxybenzyl alcohol. N-Bromosuccinimide (recrystallized from water prior to use, $5.41 \mathrm{~g}, 30.4 \mathrm{mmol}, 1.05$ equiv) was added to a solution of 2-chloro-3methoxybenzyl alcohol $(5.00 \mathrm{~g}, 29.0 \mathrm{mmol}, 1$ equiv) in dimethylformamide $(25 \mathrm{~mL})$. After stirring at $23{ }^{\circ} \mathrm{C}$ in the dark for $56 \mathrm{~h}$, the reaction mixture was partitioned between water $(600$ $\mathrm{mL})$ and ether $(300 \mathrm{~mL})$. The aqueous layer was separated and extracted with ether $(2 \times 300 \mathrm{~mL})$. The organic layers were combined and washed with an aqueous solution of sodium hydroxide $(1 \mathrm{~N}, 2 \times 100 \mathrm{~mL})$. The washed organic solution was dried over anhydrous sodium sulfate. The dried solution was filtered and the filtrate was concentrated. The residue was recrystallized (ethyl acetate-hexanes) to afford 6-bromo-2-chloro-3-methoxybenzyl alcohol as a white solid, mp 92$93{ }^{\circ} \mathrm{C}(6.70 \mathrm{~g}, 92 \%)$.

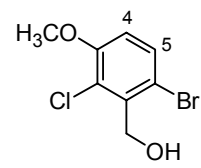

${ }^{1} \mathrm{H}$ NMR (500 MHz, CDCl 3 ) $\delta: \quad 7.44$ (d, J=9.0 Hz, 1H, Ar-H), 6.78 (d, J=8.5 Hz, 1H, Ar-H), 4.97 (d, J=7.0 Hz, 2H, C(1)-CH $\left.\mathbf{H}_{2} \mathrm{OH}\right), 3.88$ (s, 3H, C(2)$\left.\mathrm{OCH}_{3}\right), 2.25\left(\mathrm{t}, \mathrm{J}=7.0 \mathrm{~Hz}, 1 \mathrm{H}, \mathrm{C}(1)-\mathrm{CH}_{2} \mathrm{OH}\right)$

${ }^{13} \mathrm{C}$ NMR $\left(100 \mathrm{MHz}, \mathrm{CDCl}_{3}\right) \delta: \quad 154.9,138.0,131.4,124.3,115.7,112.7,63.0,56.5$

$\operatorname{HRMS}\left(\mathrm{EI}^{+}\right)$:

$m / z$ calcd for $\left(\mathrm{C}_{8} \mathrm{H}_{8} \mathrm{BrClO}_{2}\right)^{+} 249.9396$, found 249.9402 
FTIR $\left(\mathrm{cm}^{-1}\right)$ :

$3390,2941,2899,2834,1567,1462,1434,1289,1274$,

$1069,1013,917,802$

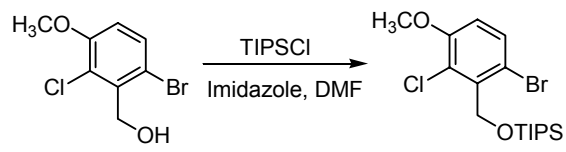

6-Bromo-2-chloro-3-methoxybenzyl alcohol triisopropylsilyl ether. Triisopropylsilyl chloride ( $8.34 \mathrm{~mL}, 39.0 \mathrm{mmol}, 2.0$ equiv) was added via syringe over $2 \mathrm{~min}$ to a solution of 6-bromo-2chloro-3-methoxybenzyl alcohol (4.90 g, $19.5 \mathrm{mmol}, 1$ equiv) and imidazole ( $4.69 \mathrm{~g}, 78.0 \mathrm{mmol}$, 4.0 equiv) in dimethylformamide $(40 \mathrm{~mL})$ at $23{ }^{\circ} \mathrm{C}$. The resulting solution was stirred at $23{ }^{\circ} \mathrm{C}$ for $3 \mathrm{~h}$, then was diluted with ether $(200 \mathrm{~mL})$. The diluted product mixture was washed with water $(2 \times 100 \mathrm{~mL})$ and the combined aqueous layers were extracted with ether $(2 \times 200 \mathrm{~mL})$. The organic layers were combined and dried over anhydrous sodium sulfate. The dried solution was filtered and the filtrate was concentrated. The residue was purified by flash column chromatography (5\% ether-hexanes) to afford the triisopropylsilyl ether as a white solid ( $7.63 \mathrm{~g}$, $96 \%)$.

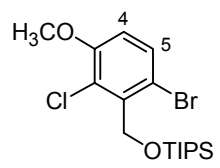

$\left.{ }^{1} \mathrm{H} \mathrm{NMR} \mathrm{(500} \mathrm{MHz,} \mathrm{CDCl}\right)$ ) $: \quad 7.44$ (d, J=9.0 Hz, 1H, Ar-H), 6.76 (d, J=9.0 Hz, 1H, Ar-H), 5.05 (s, 2H, C(1)-CH $\left.\mathbf{H}_{2} \mathrm{OTIPS}\right), 3.89$ (s, 3H, C(3)-OCH ${ }_{3}$ ), $1.17\left(\mathrm{~m}, 3 \mathrm{H}, \mathrm{C}(1)-\mathrm{CH}_{2} \mathrm{OSi}\left(\mathrm{CH}\left(\mathrm{CH}_{3}\right)_{2}\right)_{3}\right), 1.11(\mathrm{~d}, \mathrm{~J}=2.0 \mathrm{~Hz}$, $\left.18 \mathrm{H}, \mathrm{C}(1)-\mathrm{CH}_{2} \mathrm{OSi}\left(\mathrm{CH}\left(\mathrm{CH}_{3}\right)_{2}\right)_{3}\right)$

${ }^{13} \mathrm{C}$ NMR $\left(100 \mathrm{MHz}, \mathrm{CDCl}_{3}\right) \delta: \quad 154.7,138.4,131.2,124.8,116.3,112.3,63.7,56.4,18.0$ 12.2 
$\operatorname{HRMS}\left(\mathrm{CI}^{+}\right)$:

$\operatorname{FTIR}\left(\mathrm{cm}^{-1}\right)$ : $m / z$ calcd for $\left(\mathrm{C}_{17} \mathrm{H}_{28} \mathrm{BrClO}_{2} \mathrm{Si}+\mathrm{NH}_{4}\right)^{+}$424.1074, found 424.1069

$2941,2864,1567,1462,1433,1280,1073$

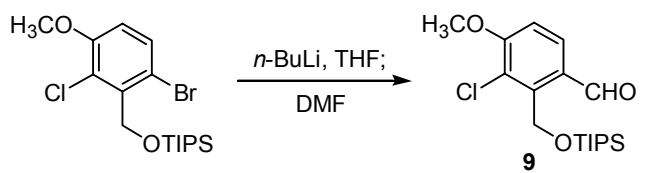

Aldehyde 9. A solution of tert-butyllithium in pentanes (1.78 M, $26.4 \mathrm{~mL}, 47.0 \mathrm{mmol}, 3.0$ equiv) was added via syringe over $25 \mathrm{~min}$ to a deoxygenated solution of the triisopropylsilyl ether (6.39 g, $15.7 \mathrm{mmol}$, 1 equiv) in tetrahydrofuran $(400 \mathrm{~mL})$ at $-96{ }^{\circ} \mathrm{C}$ (liquid $\mathrm{N}_{2} /$ ethanol cooling bath). The resulting yellow solution was stirred at $-96{ }^{\circ} \mathrm{C}$ for $5 \mathrm{~min}$. Dimethylformamide $(3.64 \mathrm{~mL}$, $47.0 \mathrm{mmol}, 3.0$ equiv) was added, causing the reaction mixture to turn colorless. After $10 \mathrm{~min}$ at $-96{ }^{\circ} \mathrm{C}$, the cooling bath was removed and the reaction mixture was allow to warm to $23{ }^{\circ} \mathrm{C}$ over approximately $1 \mathrm{~h}$. Saturated aqueous ammonium chloride solution (300 mL) was added, and tetrahydrofuran was removed in vacuo. The aqueous residue was extracted with ether $(3 \times 200$ $\mathrm{mL}$ ). The combined organic layers were dried over anhydrous sodium sulfate. The dried solution was filtered and the filtrate was concentrated. The residue was purified by flash column chromatography (20\% ether-hexanes) to afford aldehyde 9 as a colorless oil (5.03 g, 90\%).

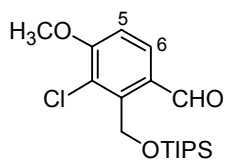

${ }^{1} \mathrm{H}$ NMR $\left(500 \mathrm{MHz}, \mathrm{CDCl}_{3}\right) \delta: \quad 10.41(\mathrm{~s}, 1 \mathrm{H}, \mathrm{C}(1)-\mathrm{C}(\mathrm{O}) \mathbf{H}), 7.90$ (d, J=9.0 Hz, 1H, C(6)-H), $6.96(\mathrm{~d}, \mathrm{~J}=9.0 \mathrm{~Hz}, 1 \mathrm{H}, \mathrm{C}(5)-\mathbf{H}), 5.31(\mathrm{~s}, 2 \mathrm{H}, \mathrm{C}(2)-$ $\mathrm{CH}_{2} \mathrm{OTIPS}$ ), 3.97 (s, 3H, C(4)-OCH 3 ), 1.15 (m, 3H, C(2)$\left.\left.\mathrm{CH}_{2} \mathrm{OSi}\left(\mathrm{CH}\left(\mathrm{CH}_{3}\right)_{2}\right)_{3}\right)\right), 1.04(\mathrm{~d}, \mathrm{~J}=7.5 \mathrm{~Hz}, 18 \mathrm{H}, \mathrm{C}(2)-$ $\left.\mathrm{CH}_{2} \mathrm{OSi}\left(\mathrm{CH}\left(\mathrm{CH}_{3}\right)_{2}\right)_{3}\right)$ 
${ }^{13} \mathrm{C}$ NMR $\left(100 \mathrm{MHz}, \mathrm{CDCl}_{3}\right) \delta: \quad 190.6,158.8,142.1,129.4,128.6,122.1,110.4,59.0,56.5$, $17.9,11.9$

HRMS $\left(\mathrm{ESI}^{+}\right)$:

$m / z$ calcd for $\left(\mathrm{C}_{18} \mathrm{H}_{29} \mathrm{ClO}_{3} \mathrm{Si}+\mathrm{H}\right)^{+}$357.1653, found 357.1645

FTIR $\left(\mathrm{cm}^{-1}\right)$ :

$2943,2866,1686,1586,1282,1061$

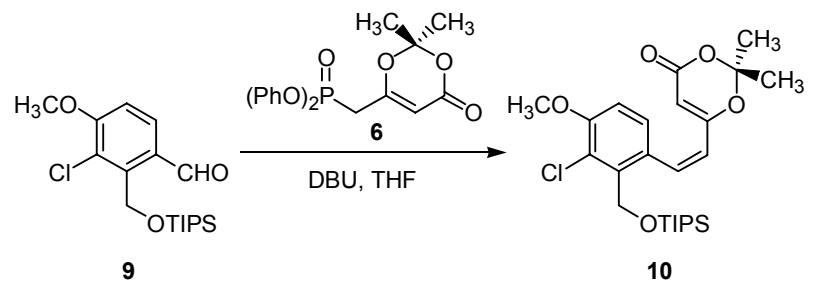

(Z)-Olefin 10. DBU (1.27 mL, $8.46 \mathrm{mmol}, 2.0$ equiv) was added dropwise via syringe over 2 min to a solution of phosphonate $6(1.74 \mathrm{~g}, 4.65 \mathrm{mmol}, 1.1$ equiv) in tetrahydrofuran $(16 \mathrm{~mL})$ at $0{ }^{\circ} \mathrm{C}$. After stirring for $10 \mathrm{~min}$ at $0{ }^{\circ} \mathrm{C}$, the resulting mixture was cooled to $-78^{\circ} \mathrm{C}$. A solution of aldehyde 9 (1.51 g, $4.23 \mathrm{mmol}, 1$ equiv) in tetrahydrofuran $(16 \mathrm{~mL})$ was added over 2 min via cannula. The resulting mixture was allowed to warm to $0{ }^{\circ} \mathrm{C}$ and was stirred at $0{ }^{\circ} \mathrm{C}$ for $15 \mathrm{~h}$. Saturated aqueous ammonium chloride solution $(60 \mathrm{~mL})$ and water $(10 \mathrm{~mL})$ were added, and the resulting biphasic mixture was extracted with ether $(4 \times 75 \mathrm{~mL})$. The organic layers were combined and dried over anhydrous sodium sulfate. The dried solution was filtered and the filtrate was concentrated. The residue was purified by flash column chromatography $(50 \%$ ether-hexanes) to afford the (Z)-olefin 10 as a white solid (1.51 g, 74\%).

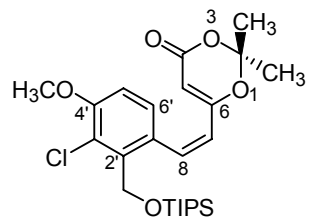

${ }^{1} \mathrm{H}$ NMR (500 MHz, CDCl $)$ ): 7.20 (d, J=12.0 Hz, 1H, C(8)-H), 7.12 (d, J=8.5 Hz, 1H, C(6')-H), 6.81 (d, J=8.0 Hz, 1H, C(5')-H), 5.97 (d, J=12.0 
$\mathrm{Hz}, 1 \mathrm{H}, \mathrm{C}(7)-\mathbf{H}), 5.27$ (s, 1H, C(5)-H), 4.93 (s, 2H, C(2')$\mathrm{CH}_{2} \mathrm{OTIPS}$ ), 3.91 (s, 3H, C(4')-OCH 3 ), 1.49 (s, 6H, C(2)$\left.\left(\mathrm{CH}_{3}\right)_{2}\right), 1.14\left(\mathrm{~m}, 3 \mathrm{H}, \mathrm{C}(2)-\mathrm{CH}_{2} \mathrm{OSi}\left(\mathrm{CH}\left(\mathrm{CH}_{3}\right)_{2}\right)_{3}\right), 1.06(\mathrm{~d}$, $\left.\mathrm{J}=7.5 \mathrm{~Hz}, 18 \mathrm{H}, \mathrm{C}(2)-\mathrm{CH}_{2} \mathrm{OSi}\left(\mathrm{CH}\left(\mathrm{CH}_{3}\right)_{2}\right)_{3}\right)$

${ }^{13} \mathrm{C}$ NMR $\left(100 \mathrm{MHz}, \mathrm{CDCl}_{3}\right) \delta: \quad 163.7,161.7,154.9,139.5,137.4,130.1,128.5,122.3$, $121.5,109.8,106.0,95.9,61.0,56.3,24.8,18.0,12.0$

HRMS (ESI $\left.{ }^{+}\right): \quad m / z$ calcd for $\left(\mathrm{C}_{25} \mathrm{H}_{37} \mathrm{ClO}_{5} \mathrm{Si}+\mathrm{H}\right)^{+}$481.2177, found 481.2174

FTIR $\left(\mathrm{cm}^{-1}\right): \quad 2942,2863,1733,1718,1560,1275,1060$

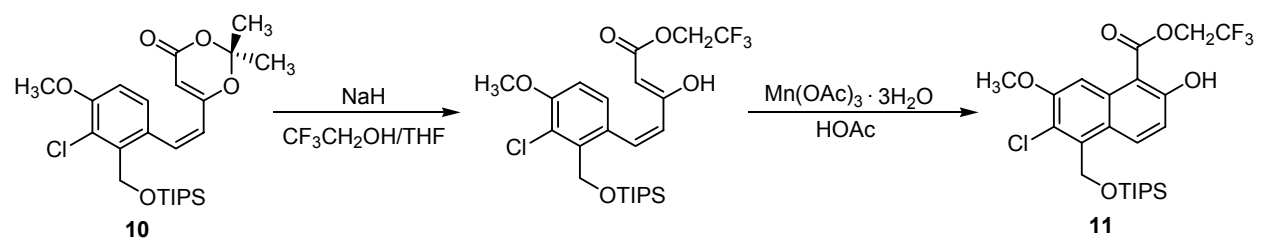

Trifluoroethyl 2-hydroxy-1-naphthoic acid ester 11. Sodium hydride $(60 \%$ dispersion in mineral oil, $27.0 \mathrm{mg}, 1.13 \mathrm{mmol}, 4.3$ equiv) was added to a solution of trifluoroethanol (150 $\mu \mathrm{L}$, $2.06 \mathrm{mmol}, 7.9$ equiv) in tetrahydrofuran $(1.5 \mathrm{~mL})$. The resulting mixture was stirred at $23{ }^{\circ} \mathrm{C}$ for 20 min. (Z)-Olefin 10 (125 mg, $0.260 \mathrm{mmol}, 1$ equiv) was added, and the resulting yellow solution was stirred at $23{ }^{\circ} \mathrm{C}$ for $1 \mathrm{~h}$. The reaction mixture was concentrated in vacuo, and the residue was taken up in acetic acid $(3.0 \mathrm{~mL})$. Manganese (III) acetate dihydrate (139 mg, 0.520 mmol, 2.0 equiv) was added. After stirring for $2 \mathrm{~h}$ at $40{ }^{\circ} \mathrm{C}$, the resulting mixture was partitioned between water $(20 \mathrm{~mL})$ and ethyl acetate $(25 \mathrm{~mL})$. The aqueous layer was separated and extracted further with ethyl acetate $(2 \times 25 \mathrm{~mL})$. The organic layers were combined and washed with saturated aqueous sodium bicarbonate solution $(2 \times 25 \mathrm{~mL})$. The washed organic solution was dried over anhydrous sodium sulfate. The dried solution was filtered and the filtrate was concentrated. The residue was purified by flash column chromatography (14\% ether-hexanes) to 
give trifluoroethyl 2-hydroxy-1-naphthoic acid ester 11 as a yellow solid (125 mg, 93\% over 2 steps).

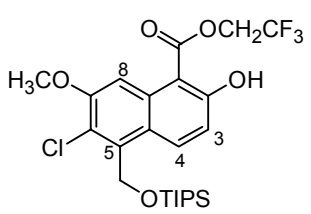

${ }^{1} \mathrm{H}$ NMR (500 MHz, $\left.\mathrm{CDCl}_{3}\right) \delta: \quad 11.83$ (s, 1H, C(2)-OH), 8.47 (d, J=9.0 Hz, 1H, C(4)-H), 8.17 (s, 1H, C(8)-H), 7.10 (d, J=9.5 Hz, 1H, C(3)-H), 5.36 (s, 2H, C(5)-CH $\left.\mathbf{H}_{2} \mathrm{OTIPS}\right), 4.85$ (q, J=7.0 Hz, 2H, C(1)$\left.\mathrm{C}(\mathrm{O}) \mathrm{OCH}_{2} \mathrm{CF}_{3}\right), 3.99$ (s, 3H , C(7)-OCH $), 1.18(\mathrm{~m}, 3 \mathrm{H}$, $\left.\mathrm{C}(5)-\mathrm{CH}_{2} \mathrm{OSi}\left(\mathrm{CH}\left(\mathrm{CH}_{3}\right)_{2}\right)_{3}\right), 1.06(\mathrm{~d}, \mathrm{~J}=7.0 \mathrm{~Hz}, 18 \mathrm{H}, \mathrm{C}(5)-$ $\left.\mathrm{CH}_{2} \mathrm{OSi}\left(\mathrm{CH}\left(\mathrm{CH}_{3}\right)_{2}\right)_{3}\right)$

${ }^{13} \mathrm{C}$ NMR $\left(100 \mathrm{MHz}, \mathrm{CDCl}_{3}\right) \delta: \quad 170.5,165.5,154.8,136.1,135.4,132.1,123.5,123.2$ (q, $\mathrm{J}=275 \mathrm{~Hz}), 121.3,117.1,105.6,102.9,61.6(\mathrm{q}, \mathrm{J}=36.6 \mathrm{~Hz})$, $60.3,56.0,18.0,12.1$

$\operatorname{HRMS}\left(\mathrm{ESI}^{-}\right)$: $\quad \mathrm{m} / \mathrm{z}$ calcd for $\left(\mathrm{C}_{24} \mathrm{H}_{32} \mathrm{ClF}_{3} \mathrm{O}_{5} \mathrm{Si}-\mathrm{H}\right)^{-}$519.1582, found 519.1599

$\operatorname{FTIR}\left(\mathrm{cm}^{-1}\right)$ : 2943, 2867, 1658, 1610, 1592, 1464, 1418, 1315, 1281, $1201,1175,1110,1050$

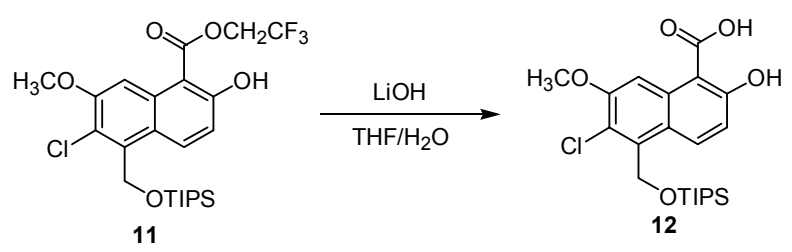

2-Hydroxy-1-naphthoic acid 12. Lithium hydroxide monohydrate (302 mg, $7.20 \mathrm{mmol}, 30$ equiv) was added to a solution of trifluoroethyl 2-hydroxy-1-naphthoic acid ester 11 (125 mg, 
$0.240 \mathrm{mmol}, 1$ equiv) in tetrahydrofuran $(8 \mathrm{~mL})$ and water $(4 \mathrm{~mL})$. The resulting biphasic mixture was allowed to stir at $40{ }^{\circ} \mathrm{C}$ for $18 \mathrm{~h}$. Water $(10 \mathrm{~mL})$ was added, the biphasic mixture was cooled to $0{ }^{\circ} \mathrm{C}$, and $2 \mathrm{~N}$ aqueous hydrochloric acid solution was carefully added to an end point of $\mathrm{pH} \sim 1.0(\sim 3.5 \mathrm{~mL})$. The product was extracted with ethyl acetate $(3 \times 20 \mathrm{~mL})$. The combined organic layers were washed with brine $(2 \times 12 \mathrm{~mL})$. The washed organic solution was dried over anhydrous sodium sulfate. The dried solution was filtered and the filtrate was concentrated to give crude acid $\mathbf{1 2}$ as a white solid (105 mg, 100\%).

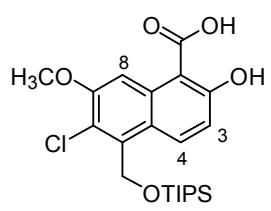

${ }^{1} \mathrm{H}$ NMR (500 MHz, CDCl $)$ ) : 12.08 (s, br, 1H, C(2)-OH), 8.49 (d, J=10.0 Hz, 1H, C(4)H), $8.41(\mathrm{~s}, 1 \mathrm{H}, \mathrm{C}(8)-\mathbf{H}), 7.11(\mathrm{~d}, \mathrm{~J}=9.5 \mathrm{~Hz}, 1 \mathrm{H}, \mathrm{C}(3)-\mathbf{H})$, 5.38 (s, 2H, C(5)-CH $\mathbf{H}_{2} \mathrm{OTIPS}$ ), 4.05 (s, 3H, C(7)-OCH ${ }_{3}$ ), $1.19\left(\mathrm{~m}, 3 \mathrm{H}, \mathrm{C}(5)-\mathrm{CH}_{2} \mathrm{OSi}\left(\mathrm{CH}\left(\mathrm{CH}_{3}\right)_{2}\right)_{3}\right), 1.07(\mathrm{~d}, \mathrm{~J}=7.0 \mathrm{~Hz}$, $\left.18 \mathrm{H}, \mathrm{C}(5)-\mathrm{CH}_{2} \mathrm{OSi}\left(\mathrm{CH}\left(\mathrm{CH}_{3}\right)_{2}\right)_{3}\right)$

${ }^{13} \mathrm{C} \mathrm{NMR}\left(100 \mathrm{MHz}, \mathrm{CDCl}_{3}\right) \delta: \quad 176.4,166.0,154.7,135.9,135.7,132.8,123.6,121.3$, $117.2,106.0,102.8,60.3,56.2,18.0,12.0$

HRMS (ESI $)^{-}$:

$\mathrm{m} / \mathrm{z}$ calcd for $\left(\mathrm{C}_{22} \mathrm{H}_{31} \mathrm{ClO}_{5} \mathrm{Si}-\mathrm{H}\right)^{-}$437.1551, found 437.1554

$\operatorname{FTIR}\left(\mathrm{cm}^{-1}\right)$ : $2940,2865,1631,1441,1421,1283,1251,1234,1216$, $1116,1064,1014,882$ 
Table 1, Entry 1 (3 steps)

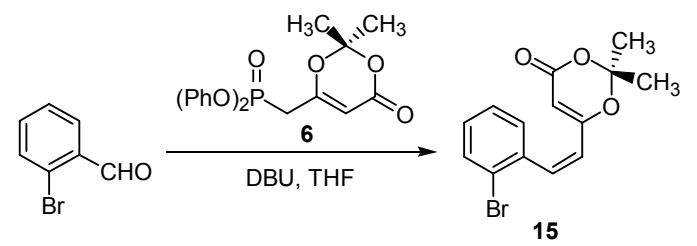

(Z)-Olefin 15. DBU ( $323 \mu \mathrm{L}, 329 \mathrm{mg}, 2.16 \mathrm{mmol}, 2.0$ equiv) was added dropwise via syringe over $2 \mathrm{~min}$ to a stirring solution of phosphonate $6(566 \mathrm{mg}, 1.51 \mathrm{mmol} 1.4$ equiv) in tetrahydrofuran $(6 \mathrm{~mL})$ at $0{ }^{\circ} \mathrm{C}$. The reaction mixture was allowed to stir at $0{ }^{\circ} \mathrm{C}$ for $20 \mathrm{~min}$. A solution of 2-bromobenzaldehyde ( $200 \mathrm{mg}, 1.08 \mathrm{mmol}, 1$ equiv) in tetrahydrofuran $(5 \mathrm{~mL})$ was added dropwise via cannula. The reaction mixture was allowed to stir at $0{ }^{\circ} \mathrm{C}$ for $18 \mathrm{~h}$. Saturated aqueous ammonium chloride solution $(20 \mathrm{~mL})$ was added, and the resulting biphasic mixture was concentrated in vacuo to remove tetrahydrofuran. The aqueous residue was extracted with ether $(3 \times 30 \mathrm{~mL})$. The organic extracts were combined and washed with brine $(2 \times 20 \mathrm{~mL})$. The washed organic solution was dried over anhydrous sodium sulfate. The dried solution was filtered and the filtrate was concentrated. The residue was purified by flash column chromatography (33\% ether-hexanes) to give (Z)-olefin 15 as a colorless oil (324 mg, $97 \%$ ).

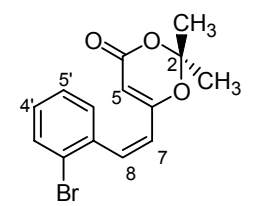

${ }^{1} \mathrm{H} \mathrm{NMR}\left(500 \mathrm{MHz}, \mathrm{CDCl}_{3}\right)$ ): 7.58 (d, J=8.0 Hz, 1H, C(3')-H ), 7.27 (d, J=4.0 Hz, 2H, $\left.\mathrm{C}\left(5^{\prime}, 6^{\prime}\right)-\mathbf{H}\right), 7.18\left(\mathrm{~m}, \mathrm{~J}_{1}=4.0 \mathrm{~Hz}, \mathrm{~J}_{2}=8.0 \mathrm{~Hz}, 1 \mathrm{H}, \mathrm{C}\left(4^{\prime}\right)-\mathbf{H}\right)$, $6.92(\mathrm{~d}, \mathrm{~J}=9.6 \mathrm{~Hz}, 1 \mathrm{H}, \mathrm{C}(8)-\mathbf{H}), 6.09(\mathrm{~d}, \mathrm{~J}=8.0 \mathrm{~Hz}, 1 \mathrm{H}$, $\mathrm{C}(7)-\mathbf{H}), 5.33$ (s, 1H, C(5)-H ), 1.46 (s, 6H, C(2)-(CH $\left.\mathrm{CH}_{3}\right)$

${ }^{13} \mathrm{C} \mathrm{NMR}\left(100 \mathrm{MHz}, \mathrm{CDCl}_{3}\right) \delta: \quad 163.0,161.5,138.9,137.1,132.0,130.5,129.5,126.4$ $123.0,122.5,106.2,96.5,24.6$

HRMS (ESI ${ }^{+}$):

$m / z$ calcd for $\left(\mathrm{C}_{14} \mathrm{H}_{12} \mathrm{BrO}_{3}+\mathrm{H}\right)^{+}$309.0126, found 309.0125 
FTIR $\left(\mathrm{cm}^{-1}\right)$ :

2997, 2942, 1732, 1643, 1596, 1577, 1468, 1433, 1391,

$1376,1329,1274,1253,1203,1018,964,905,822,744$

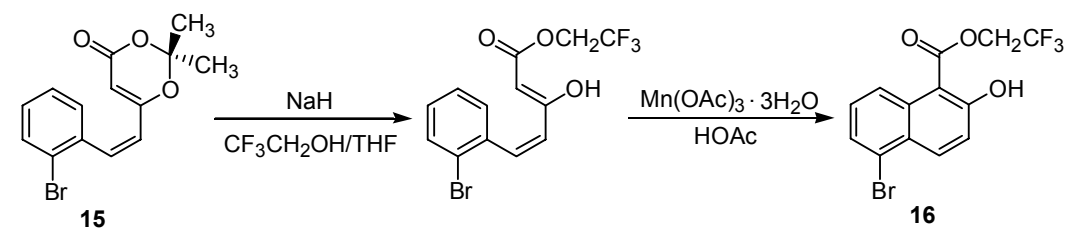

Trifluoroethyl 2-hydroxy-1-naphthoic ester 16. Sodium hydride $(60 \%$ dispersion in mineral oil, $40.3 \mathrm{mg}, 1.68 \mathrm{mmol}, 6.0$ equiv) was added to a stirring solution of trifluoroethanol ( $245 \mu \mathrm{L}$, $336 \mathrm{mg}, 3.36 \mathrm{mmol}, 10.0$ equiv) in tetrahydrofuran $(2 \mathrm{~mL})$ at $23{ }^{\circ} \mathrm{C}$. The reaction mixture was allowed to stir at $23{ }^{\circ} \mathrm{C}$ for $20 \mathrm{~min}$. A solution of (Z)-olefin $15(86.5 \mathrm{mg}, 0.280 \mathrm{mmol}, 1$ equiv) in tetrahydrofuran $(2 \mathrm{~mL})$ was added via cannula. The resulting mixture was allowed to stir at 23 ${ }^{\circ} \mathrm{C}$ for $45 \mathrm{~min}$ and was then concentrated in vacuo. The residue was taken up in acetic acid (2 $\mathrm{mL}$ ). Manganese (III) acetate dihydrate (150 mg, $0.560 \mathrm{mmol}, 2.0$ equiv) was added. After stirring for $17 \mathrm{~h}$ at $23{ }^{\circ} \mathrm{C}$, the resulting mixture was partitioned between water $(25 \mathrm{~mL})$ and ethyl acetate $(25 \mathrm{~mL})$. The aqueous layer was separated and extracted further with ethyl acetate $(2 \times$ $25 \mathrm{~mL}$ ). The organic extracts were combined and washed with saturated aqueous sodium bicarbonate solution $(2 \times 15 \mathrm{~mL})$. The washed organic solution was dried over anhydrous sodium sulfate. The dried solution was filtered and the filtrate was concentrated. The residue was purified by flash column chromatography (33\% ether-hexanes) to give trifluoroethyl 2-hydroxy1-naphthoic acid ester 16 as a white solid (78.2 mg, 80\%).

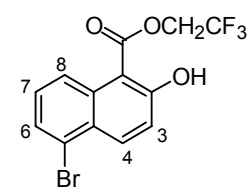

$\left.{ }^{1} \mathrm{H} \mathrm{NMR} \mathrm{(500} \mathrm{MHz,} \mathrm{CDCl}\right)$ ) $: \quad 11.69$ (s, $\left.1 \mathrm{H}, \mathrm{C}(2)-\mathrm{OH}\right), 8.63\left(\mathrm{dd}, \mathrm{J}_{1}=1.0 \mathrm{~Hz}, \mathrm{~J}_{2}=9.0 \mathrm{~Hz}, 1 \mathrm{H}\right.$, $\mathrm{C}(8)-\mathbf{H}), 8.48$ (d, J=9.0 Hz, 1H, C(4)-H), 7.70 (dd, $\mathrm{J}_{1}=1.0$ $\left.\mathrm{Hz}, \mathrm{J}_{2}=7.5 \mathrm{~Hz}, 1 \mathrm{H}, \mathrm{C}(6)-\mathbf{H}\right), 7.40\left(\mathrm{dd}, \mathrm{J}_{1}=7.5 \mathrm{~Hz}, \mathrm{~J}_{2}=9.0\right.$ 
Hz, C(7)-H), 7.27 (d, J=9.5 Hz, 1H, C(3)-H), 4.87 (q, $\left.\mathrm{J}=8.0 \mathrm{~Hz}, 2 \mathrm{H}, \mathrm{C}(1)-\mathrm{C}(\mathrm{O}) \mathrm{OCH}_{2} \mathrm{CF}_{3}\right)$

${ }^{13} \mathrm{C}$ NMR $\left(100 \mathrm{MHz}, \mathrm{CDCl}_{3}\right) \delta: \quad 170.1,165.1,136.7,133.2,129.2,128.5,127.0,124.8$ 124.1, 122.9 (q, J=275 Hz), 120.5, 103.7, 61.3 (q, J=36.7 $\mathrm{Hz}), 127.1,127.0$

HRMS (ESI-):

$m / z$ calcd for $\left(\mathrm{C}_{13} \mathrm{H}_{8} \mathrm{BrF}_{3} \mathrm{O}_{3}-\mathrm{H}\right)^{-} 346.9531$, found 346.9529

$\operatorname{FTIR}\left(\mathrm{cm}^{-1}\right)$ :

$3426,1656,1578,1463,1446,1411,1321,1281,1237$, $1171,1143,1097,1060,1002,966,830,807,751,737,705$ 
Table 1, Entry 2 (3 steps)

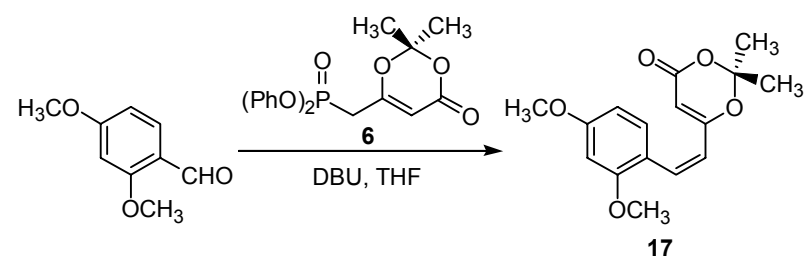

(Z)-Olefin 17. DBU (360 $\mu \mathrm{L}, 365 \mathrm{mg}, 2.4 \mathrm{mmol}, 2.0$ equiv) was added dropwise via syringe over $1 \mathrm{~min}$ to a stirring solution of phosphonate $6(631 \mathrm{mg}, 1.68 \mathrm{mmol} 1.4$ equiv) in tetrahydrofuran $(5 \mathrm{~mL})$ at $23{ }^{\circ} \mathrm{C}$. After stirring for $20 \mathrm{~min}$ at $23{ }^{\circ} \mathrm{C}$, the reaction mixture was cooled to $0{ }^{\circ} \mathrm{C}$. A solution of 2,4-dimethoxybenzaldehyde (200 mg, $1.20 \mathrm{mmol}, 1$ equiv) in tetrahydrofuran $(5 \mathrm{~mL})$ was added dropwise via cannula. The resulting mixture was allowed to stir at $0{ }^{\circ} \mathrm{C}$ for $18 \mathrm{~h}$. Saturated aqueous ammonium chloride solution $(20 \mathrm{~mL})$ was added, and the resulting biphasic mixture was concentrated in vacuo to remove tetrahydrofuran. The aqueous residue was extracted with ethyl acetate $(3 \times 30 \mathrm{~mL})$. The organic extracts were combined and washed with brine $(2 \times 20 \mathrm{~mL})$. The washed organic solution was dried over anhydrous sodium sulfate. The dried solution was filtered and the filtrate was concentrated. The residue was purified by flash column chromatography (50\% ethyl acetate-hexanes) to give (Z)-olefin $\mathbf{1 7}$ as a bright yellow solid (269 mg, 77\%).

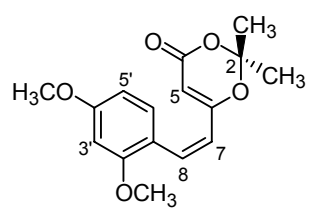

${ }^{1} \mathrm{H} \mathrm{NMR}\left(500 \mathrm{MHz}, \mathrm{CDCl}_{3}\right)$ ): 7.26 (d, J=8.5 Hz, 1H, C(6')-H ) 6.93 (d, J=12.5 Hz, 1H, C(8)-H), 6.43-6.41 (m, 2H, C(3', ', )-H), $5.84(\mathrm{~d}, \mathrm{~J}=12.5 \mathrm{~Hz}$, $1 \mathrm{H}, \mathrm{C}(7)-\mathbf{H}), 5.33$ (s, 1H, C(5)-H), 3.81 (s, 3H, - $\left.\mathrm{OCH}_{3}\right)$, $3.80\left(\mathrm{~s}, 3 \mathrm{H},-\mathrm{OCH}_{3}\right), 1.58\left(\mathrm{~s}, 6 \mathrm{H}, \mathrm{C}(2)-\left(\mathrm{CH}_{3}\right)_{2}\right)$

${ }^{13} \mathrm{C} \mathrm{NMR}\left(100 \mathrm{MHz}, \mathrm{CDCl}_{3}\right) \delta: \quad 164.3,161.9,161.7,158.3,135.8,131.6,119.5,117.3$ $105.9,103.9,97.6,94.9,55.4,55.3,24.8$ 
$\operatorname{HRMS}\left(\mathrm{ESI}^{+}\right)$:

$\operatorname{FTIR}\left(\mathrm{cm}^{-1}\right)$ : $m / z$ calcd for $\left(\mathrm{C}_{16} \mathrm{H}_{18} \mathrm{O}_{5}+\mathrm{H}\right)^{+} 291.1232$, found 291.1231

2998, 2942, 2839, 1720, 1607, 1570, 1390, 1295, 1271, $1209,1017,830$

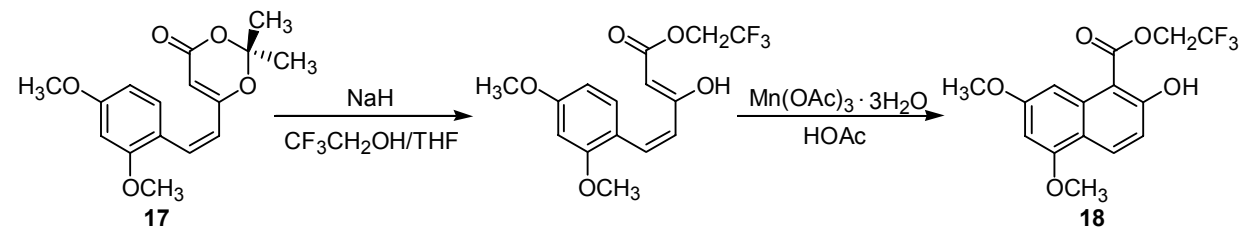

Trifluoroethyl 2-hydroxy-1-naphthoic acid ester 18. Sodium hydride $(60 \%$ dispersion in mineral oil, $98.8 \mathrm{mg}, 4.11 \mathrm{mmol}, 6.0$ equiv) was added to a stirring solution of trifluoroethanol $\left(500 \mu \mathrm{L}, 686 \mathrm{mg}, 6.86 \mathrm{mmol}, 10.0\right.$ equiv) in tetrahydrofuran $(5 \mathrm{~mL})$ at $23{ }^{\circ} \mathrm{C}$. The resulting mixture was allowed to stir at $23{ }^{\circ} \mathrm{C}$ for $20 \mathrm{~min}$. A solution of (Z)-olefin 17 (199 $\mathrm{mg}, 0.686$ mmol, 1 equiv) in tetrahydrofuran $(5 \mathrm{~mL})$ was added via cannula. After stirring for $45 \mathrm{~min}$ at 23 ${ }^{\circ} \mathrm{C}$, the resulting mixture was concentrated in vacuo. The residue was taken up in acetic acid (3 $\mathrm{mL}$ ). Manganese (III) acetate dihydrate (368mg, $1.37 \mathrm{mmol}, 2.0$ equiv) was added. After stirring for $17 \mathrm{~h}$ at $23{ }^{\circ} \mathrm{C}$, the reaction mixture was partitioned between with water $(30 \mathrm{~mL})$ and ethyl acetate $(25 \mathrm{~mL})$. The aqueous layer was separated and extracted further with ethyl acetate $(2 \times$ $25 \mathrm{~mL}$ ). The organic extracts were combined and washed with saturated aqueous sodium bicarbonate solution $(2 \times 20 \mathrm{~mL})$. The washed organic solution was dried over anhydrous sodium sulfate. The dried solution was filtered and the filtrate was concentrated. The residue was purified by flash column chromatography ( $20 \%$ ethyl acetate-hexanes) to give trifluoroethyl 2hydroxy-1-naphthoic acid ester 18 as a white solid (205 mg, $91 \%$ over two steps).

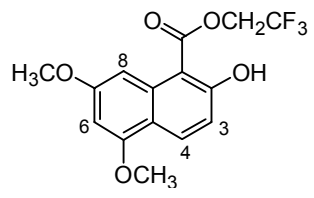

${ }^{1} \mathrm{H}$ NMR (500 MHz, $\left.\mathrm{CDCl}_{3}\right) \delta: \quad 11.85$ (s, 1H, C(2)-OH), 8.35 (d, J=9.0 Hz, 1H, C(4)-H), $7.69(\mathrm{~d}, \mathrm{~J}=2.0 \mathrm{~Hz}, 1 \mathrm{H}, \mathrm{C}(8)-\mathbf{H}), 6.96(\mathrm{~d}, \mathrm{~J}=9.5 \mathrm{~Hz}, 1 \mathrm{H}$, 
$\mathrm{C}(3)-\mathbf{H}), 6.42$ (d, J=2.5 Hz, 1H, C(6)-H), 4.83 (q, J=8.5 Hz, $\left.2 \mathrm{H}, \mathrm{C}(1)-\mathrm{C}(\mathrm{O}) \mathrm{OCH}_{2} \mathrm{CF}_{3}\right), 3.94\left(\mathrm{~s}, 3 \mathrm{H},-\mathrm{OCH}_{3}\right), 3.90(\mathrm{~s}, 3 \mathrm{H}$, $\left.-\mathrm{OCH}_{3}\right)$

${ }^{13} \mathrm{C}$ NMR $\left(100 \mathrm{MHz}, \mathrm{CDCl}_{3}\right) \delta: \quad 171.0,166.3,161.3,157.1,134.2,131.8,123.2$ (q, J=275

$\mathrm{Hz}), 116.1,115.1,102.8,97.2,95.9,61.4$ (q, J=36.4 Hz), 55.6, 55.2

$\operatorname{HRMS}\left(\mathrm{ESI}^{+}\right)$:

$m / z$ calcd for $\left(\mathrm{C}_{15} \mathrm{H}_{13} \mathrm{~F}_{3} \mathrm{O}_{5}+\mathrm{H}\right)^{+} 331.0793$, found 331.0790

$\operatorname{FTIR}\left(\mathrm{cm}^{-1}\right)$ :

2959, 2912, 2850, 1658, 1598, 1452, 1436, 1404, 1344, $1312,1252,1155,1053,955,826,777,625$ 
Table 1, Entry 3 (3 steps)

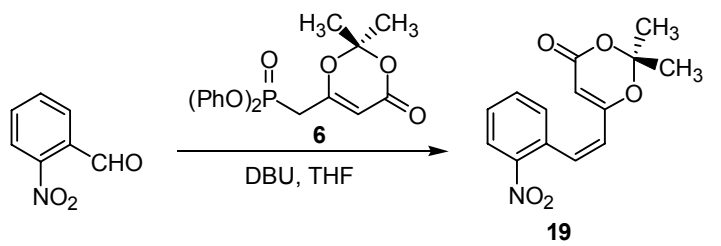

(Z)-Olefin 19. DBU (395 $\mu \mathrm{L}, 401.9 \mathrm{mg}, 2.64 \mathrm{mmol}, 2.0$ equiv) was added dropwise via syringe over $1 \mathrm{~min}$ to a stirring solution of phosphonate $6(692 \mathrm{mg}, 1.85 \mathrm{mmol}, 1.4$ equiv) in tetrahydrofuran $(5 \mathrm{~mL})$ at $23{ }^{\circ} \mathrm{C}$. After stirring for $20 \mathrm{~min}$ at $23{ }^{\circ} \mathrm{C}$, the reaction mixture was cooled to $0{ }^{\circ} \mathrm{C}$. A solution of 2-nitrobenzaldehyde (200 mg, $1.08 \mathrm{mmol}, 1$ equiv) in tetrahydrofuran $(5 \mathrm{~mL})$ was added dropwise via cannula. The reaction mixture was allowed to stir at $0{ }^{\circ} \mathrm{C}$ for $30 \mathrm{~min}$. Saturated aqueous ammonium chloride solution $(20 \mathrm{~mL})$ was added, and the resulting biphasic mixture was concentrated in vacuo to remove tetrahydrofuran. The aqueous residue was extracted with ethyl acetate $(3 \times 30 \mathrm{~mL})$. The organic extracts were combined and washed with brine $(2 \times 20 \mathrm{~mL})$. The washed solution was dried over anhydrous sodium sulfate. The dried solution was filtered and the filtrate was concentrated. The residue was purified by flash column chromatography (66\% ether-hexanes) to give (Z)-olefin 19 as a yellow oil (309 mg, 84\%).

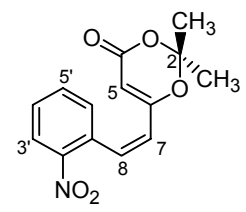

${ }^{1} \mathrm{H} \mathrm{NMR}\left(500 \mathrm{MHz}, \mathrm{CDCl}_{3}\right) \delta: \quad 8.08\left(\mathrm{dd}, \mathrm{J}_{1}=1.5 \mathrm{~Hz}, \mathrm{~J}_{2}=8.5 \mathrm{~Hz}, 1 \mathrm{H}, \mathrm{C}\left(3^{\prime}\right)-\mathbf{H}\right), 7.57(\mathrm{dt}$, $\left.\mathrm{J}_{1}=1.5 \mathrm{~Hz}, \mathrm{~J}_{2}=8.0 \mathrm{~Hz}, 1 \mathrm{H}, \mathrm{C}\left(5^{\prime}\right)-\mathbf{H}\right), 7.47\left(\mathrm{dt}, \mathrm{J}_{1}=1.0 \mathrm{~Hz}\right.$, $\left.\mathrm{J}_{2}=7.8 \mathrm{~Hz}, 1 \mathrm{H}, \mathrm{C}\left(4^{\prime}\right)-\mathbf{H}\right), 7.29\left(\mathrm{~d}, \mathrm{~J}=7.5 \mathrm{~Hz}, 1 \mathrm{H}, \mathrm{C}\left(6^{\prime}\right)-\mathbf{H}\right)$, $7.20(\mathrm{~d}, \mathrm{~J}=12.0 \mathrm{~Hz}, 1 \mathrm{H}, \mathrm{C}(8)-\mathbf{H}), 6.10$ (d, J=12.0 Hz, 1H, $\mathrm{C}(7)-\mathbf{H}), 5.28(\mathrm{~s}, 1 \mathrm{H}, \mathrm{C}(5)-\mathbf{H}), 1.27\left(\mathrm{~s}, 6 \mathrm{H}, \mathrm{C}(2)-\left(\mathrm{CH}_{3}\right)_{2}\right)$ 
${ }^{13} \mathrm{C}$ NMR $\left(100 \mathrm{MHz}, \mathrm{CDCl}_{3}\right) \delta: \quad 162.4,161.0,147.0,136.2,133.0,132.6,130.8,128.6$ $124.0,122.3,106.0,96.7,24.2$

HRMS $\left(\mathrm{ESI}^{+}\right)$:

$\mathrm{m} / \mathrm{z}$ calcd for $\left(\mathrm{C}_{14} \mathrm{H}_{13} \mathrm{NO}_{5}+\mathrm{H}\right)^{+} 276.0872$, found 276.0885

$\operatorname{FTIR}\left(\mathrm{cm}^{-1}\right)$ : 3092, 2997, 2943, 2859, 1721, 1645, 1612, 1580, 1523, $1391,1376,1345,1274,1202,1018,905,823,719$

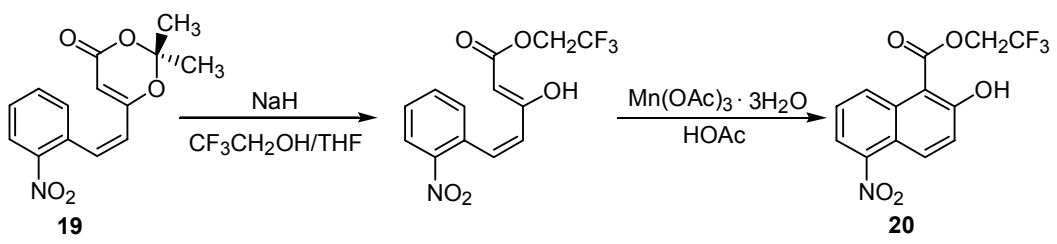

Trifluoroethyl 2-hydroxy-1-naphthoic acid ester 20. Sodium hydride $(60 \%$ dispersion in mineral oil, $16.9 \mathrm{mg}, 0.706 \mathrm{mmol}, 6.0$ equiv) was added to a stirring solution of trifluoroethanol $(85.7 \mu \mathrm{L}, 118 \mathrm{mg}, 1.12 \mathrm{mmol}, 10.0$ equiv) in tetrahydrofuran $(3 \mathrm{~mL})$. The reaction mixture was allowed to stir at $23{ }^{\circ} \mathrm{C}$ for $20 \mathrm{~min}$. A solution of (Z)-olefin 19 (32.4 mg, $0.117 \mathrm{mmol}, 1$ equiv) in tetrahydrofuran $(3 \mathrm{~mL})$ was added via cannula at $23{ }^{\circ} \mathrm{C}$. After stirring for $45 \mathrm{~min}$ at $23{ }^{\circ} \mathrm{C}$, the reaction mixture was concentrated in vacuo. The residue was taken up with acetic acid $(3 \mathrm{~mL})$. Manganese (III) acetate dihydrate ( $62.7 \mathrm{mg}, 0.234 \mathrm{mmol}, 2.0$ equiv) was added. After stirring for $2 \mathrm{~h}$ at $40{ }^{\circ} \mathrm{C}$, the reaction mixture was partitioned between water $(20 \mathrm{~mL})$ and ethyl acetate $(25$ $\mathrm{mL})$. The aqueous layer was separated and further extracted ethyl acetate $(2 \times 25 \mathrm{~mL})$. The organic extracts were combined and washed with saturated aqueous sodium bicarbonate solution $(2 \times 20 \mathrm{~mL})$. The washed organic solution was dried over anhydrous sodium sulfate. The dried solution was filtered and the filtrate was concentrated. The residue was purified by flash column chromatography (33\% ether-hexanes) to give trifluoroethyl 2-hydroxy-1-naphthoic acid ester $\mathbf{2 0}$ as a white solid ( $34.8 \mathrm{mg}, 94 \%$ over two steps).

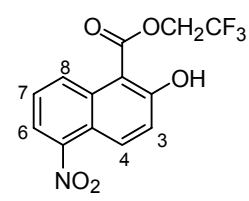


${ }^{1} \mathrm{H}$ NMR (500 MHz, CDCl $)$ ) $: \quad 11.71$ (s, $\left.1 \mathrm{H}, \mathrm{C}(2)-\mathrm{OH}\right), 8.94$ (d, J=9.0 Hz, 1H, C(8)-H), $8.54(\mathrm{~d}, \mathrm{~J}=9.5 \mathrm{~Hz}, 1 \mathrm{H}, \mathrm{C}(4)-\mathbf{H}), 7.99$ (dd, $\mathrm{J}_{1}=1.0 \mathrm{~Hz}, \mathrm{~J}_{2}=8.0$ $\mathrm{Hz}, 1 \mathrm{H}, \mathrm{C}(6)-\mathrm{H}), 7.66$ (dt, $\mathrm{J}_{1}=8.0 \mathrm{~Hz}, \mathrm{~J}_{2}=9.0 \mathrm{~Hz}, 1 \mathrm{H}, \mathrm{C}(7)-$ H), 7.39 (d, J=9.5 Hz, 1H, C(3)-H), 4.91 (q, J=8.5 Hz, 2H, $\left.\mathrm{C}(1)-\mathrm{C}(\mathrm{O}) \mathrm{OCH}_{2} \mathrm{CF}_{3}\right)$

${ }^{13} \mathrm{C}$ NMR $\left(100 \mathrm{MHz}, \mathrm{CDCl}_{3}\right) \delta: \quad 169.8,165.1,148.2,132.8,131.6,130.3,127.4,122.8(\mathrm{q}$, $\mathrm{J}=276 \mathrm{~Hz}), 122.5,121.0,120.4,104.0,61.5(\mathrm{q}, \mathrm{J}=36.8 \mathrm{~Hz})$

HRMS (ESI $\left.{ }^{-}\right)$:

$m / z$ calcd for $\left(\mathrm{C}_{13} \mathrm{H}_{8} \mathrm{~F}_{3} \mathrm{NO}_{3}-\mathrm{H}\right)^{-} 314.0276$, found 314.0279

$\operatorname{FTIR}\left(\mathrm{cm}^{-1}\right)$ :

$3021,2361,1662,1524,1343,1284,1213,1155,984,961$, 820 
Table 1, Entry 4 (3 steps)

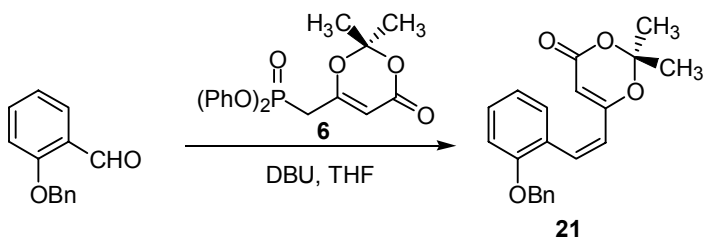

(Z)-Olefin 21. DBU ( $281 \mu \mathrm{L}, 287 \mathrm{mg}, 1.88 \mathrm{mmol}, 2.0$ equiv) was added dropwise via syringe over $1 \mathrm{~min}$ to a stirring solution of phosphonate 6 (494 $\mathrm{mg}, 1.32 \mathrm{mmol}, 1.4$ equiv) in tetrahydrofuran $(5 \mathrm{~mL})$ at $23{ }^{\circ} \mathrm{C}$. After stirring for $20 \mathrm{~min}$ at $23{ }^{\circ} \mathrm{C}$, the reaction mixture was cooled to $0{ }^{\circ} \mathrm{C}$. A solution of 2-benzyloxybenzaldehyde (200 mg, $0.942 \mathrm{mmol}, 1$ equiv) in tetrahydrofuran $(5.0 \mathrm{~mL})$ was added and the resulting mixture was allowed to stir at $0{ }^{\circ} \mathrm{C}$ for $1 \mathrm{~h}$. Saturated aqueous ammonium chloride solution $(20 \mathrm{~mL})$ was added, and the resulting biphasic mixture was concentrated in vacuo to remove tetrahydrofuran. The aqueous residue was extracted with ethyl acetate $(3 \times 30 \mathrm{~mL})$. The combined organic extracts were washed with brine $(2 \times 20 \mathrm{~mL})$. The washed organic solution was dried over anhydrous sodium sulfate. The dried solution was filtered and the filtrate was concentrated. The residue was purified by flash column chromatography (33\% ether-hexanes) to give a $(Z) /(E)$ mixture of olefin 21 (317 $\mathrm{mg}, 83 \%)$, which was used in the next reaction without further purification.

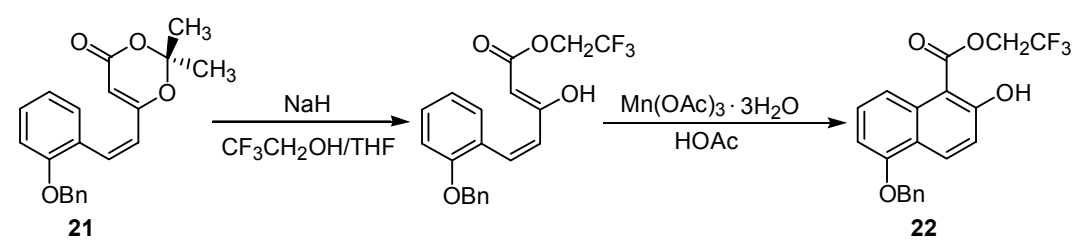

Trifluoroethyl-2-hydroxy-1-naphthoic acid ester 22. Sodium hydride $(60 \%$ dispersion in mineral oil, $136 \mathrm{mg}, 5.66 \mathrm{mmol}, 6.0$ equiv) was added to a stirring solution of trifluoroethanol (687 $\mu \mathrm{L}, 943 \mathrm{mg}, 9.43 \mathrm{mmol}, 10.0$ equiv) in tetrahydrofuran $(5 \mathrm{~mL})$ at $23{ }^{\circ} \mathrm{C}$. The reaction mixture was allowed to stir at $23^{\circ} \mathrm{C}$ for $20 \mathrm{~min}$. A $(Z) /(E)$ mixture of olefin $21(317 \mathrm{mg}, 0.943$ mmol, 1 equiv, $\sim 10: 1 \mathrm{Z}: E$, from the experiment above $)$ in tetrahydrofuran $(5 \mathrm{~mL})$ was added via cannula. After stirring for $1 \mathrm{~h}$ at $23{ }^{\circ} \mathrm{C}$, the reaction mixture was concentrated in vacuo. The 
residue was taken up in acetic acid (4 mL). Manganese (III) acetate dihydrate (506 mg, 1.89 mmol, 2.0 equiv) was added. After stirring for $1 \mathrm{~h}$ at $40{ }^{\circ} \mathrm{C}$, the reaction mixture was partitioned between with water $(20 \mathrm{~mL})$ and ethyl acetate $(30 \mathrm{~mL})$. The aqueous layer was separated and extracted further with ethyl acetate $(2 \times 30 \mathrm{~mL})$. The organic extracts were combined and washed with saturated aqueous sodium bicarbonate solution $(2 \times 20 \mathrm{~mL})$. The washed organic solution was dried over anhydrous sodium sulfate. The dried solution was filtered and the filtrate was concentrated. The residue was purified by flash column chromatography (33\% ethyl acetate-hexanes) to give trifluoroethyl 2-hydroxy-1-naphthoic acid ester 22 as a white solid (266 mg, $75 \%$ over 3 steps).

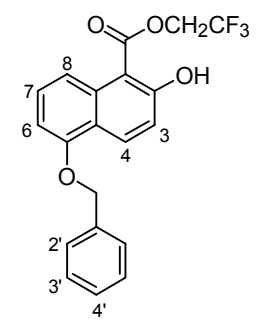

${ }^{1} \mathrm{H}$ NMR (500 MHz, $\left.\mathrm{CDCl}_{3}\right) \delta: \quad 11.76$ (s, 1H, C(2)-OH), 8.57 (d, J=9.5 Hz, 1H, C(4)-H), 8.25 (d, J=9.0 Hz, 1H, C(8)-H), 7.52-7.48 (m, 2H, C(2')-H), $7.50(\mathrm{t}, \mathrm{J}=8.5 \mathrm{~Hz}, 1 \mathrm{H}, \mathrm{C}(7)-\mathbf{H}), 7.38$ (t, J=7.5 Hz, 1H, C(4')H), 7.44 (t, J=8.0 Hz, 2H, C(3')-H), 7.14 (d, J=9.5 Hz, 1H, $\mathrm{C}(3)-\mathbf{H}), 6.86$ (d, J=8.0 Hz, 1H, C(6)-H), 5.22 (s, 2H, C(5)$\left.\mathrm{OCH}_{2} \mathrm{Ph}\right), 4.87$ (q, J=8.5 Hz, 2H, C(1)-C(O)OCH $\left.\mathrm{CF}_{3}\right)$

${ }^{13} \mathrm{C}$ NMR $\left(100 \mathrm{MHz}, \mathrm{CDCl}_{3}\right) \delta: \quad 170.7,165.4,155.1,136.7,132.9,131.9,129.4,128.6$, $128.1,127.4,123.0$ (q, J=275 Hz), 120.6, 118.0, 117.6, $104.3,103.3,70.3,61.1(\mathrm{q}, \mathrm{J}=36.7 \mathrm{~Hz})$

$\operatorname{HRMS}\left(\mathrm{ESI}^{+}\right)$: $m / z$ calcd for $\left(\mathrm{C}_{20} \mathrm{H}_{15} \mathrm{~F}_{3} \mathrm{O}_{4}+\mathrm{H}\right)^{+} 377.1000$, found 377.1007 
FTIR $\left(\mathrm{cm}^{-1}\right)$ :

$3068,3033,2974,2904,2857,1660,1620,1602,1427$, 1394, 1330, 1253, 1222, 1183, 1163, 1087, 1055, 986, 956, $909,810,792,766,744,700,662$ 
Table 1, Entry 5 (4 steps)

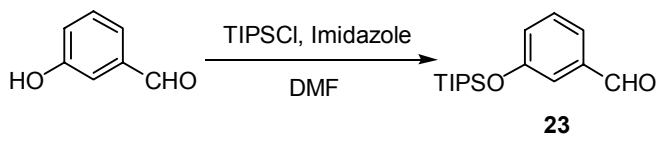

Aldehyde 23. Triisopropylsilyl chloride ( $527 \mu \mathrm{L}, 474 \mathrm{mg}, 2.46 \mathrm{mmol}, 1.5$ equiv) was added to a stirring solution of 3-hydroxybenzaldehyde (200 mg, $1.64 \mathrm{mmol}, 1$ equiv) and imidazole (295 $\mathrm{mg}, 4.91 \mathrm{mmol}, 3.0$ equiv) in dimethylformamide $(3 \mathrm{~mL})$ at $23{ }^{\circ} \mathrm{C}$. After stirring for $19 \mathrm{~h}$ at 23 ${ }^{\circ} \mathrm{C}$, the reaction mixture was diluted with ether $(50 \mathrm{~mL})$. The diluted product mixture was washed with water $(2 \times 50 \mathrm{~mL})$. The combined aqueous layers were extracted with ethyl acetate $(3 \times 20 \mathrm{~mL})$. The organic extracts were combined and washed with brine $(2 \times 25 \mathrm{~mL})$. The washed organic solution was dried over anhydrous sodium sulfate. The dried solution was filtered and the filtrate was concentrated. The residue was purified by flash column chromatography ( $10 \%$ ether-hexanes) to give aldehyde 23 as a colorless oil (398 $\mathrm{mg}, 87 \%$ ).

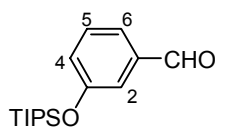

${ }^{1} \mathrm{H}$ NMR $\left(400 \mathrm{MHz}, \mathrm{CDCl}_{3}\right) \delta: \quad 9.95(\mathrm{~s}, 1 \mathrm{H}, \mathrm{C}(1)-\mathrm{C}(\mathrm{O}) \mathbf{H}), 7.45\left(\mathrm{dt}, \mathrm{J}_{1}=1.2 \mathrm{~Hz}, \mathrm{~J}_{2}=7.6 \mathrm{~Hz}\right.$, 1H, C(6)-H), 7.39 (t, J=7.6 Hz, 1H, C(5)-H), 7.36 (m, 1H, C(4)-H), 7.14 (ddd, $\mathrm{J}_{1}=0.8 \mathrm{~Hz}, \mathrm{~J}_{2}=2.4 \mathrm{~Hz}, \mathrm{~J}_{3}=8.1 \mathrm{~Hz}, 1 \mathrm{H}$, $\mathrm{C}(2)-\mathbf{H}) 1.28\left(\mathrm{~m}, 3 \mathrm{H}, \mathrm{C}(3)-\mathrm{OSi}\left(\mathrm{CH}\left(\mathrm{CH}_{3}\right)_{2}\right)_{3}\right), 1.10$ (d, $\left.\mathrm{J}=7.6 \mathrm{~Hz}, 18 \mathrm{H}, \mathrm{C}(3)-\mathrm{OSi}\left(\mathrm{CH}\left(\mathrm{CH}_{3}\right)_{2}\right)_{3}\right)$

${ }^{13} \mathrm{C}$ NMR $\left(100 \mathrm{MHz}, \mathrm{CDCl}_{3}\right) \delta: \quad 192.1,156.8,137.9,130.0,126.3,123.3,119.6,17.8,12.6$

HRMS (ESI ${ }^{+}$):

$m / z$ calcd for $\left(\mathrm{C}_{16} \mathrm{H}_{26} \mathrm{O}_{2} \mathrm{Si}+\mathrm{H}\right)^{+} 279.1780$, found 279.1778

FTIR $\left(\mathrm{cm}^{-1}\right)$ :

3396, 2946, 2868, 2724, 1704, 1598, 1584, 1484, 1458, 1446, 1387, 1311, 1280, 1250, 1167, 1140, 1074, 1003, 982, $968,883,830,790,686$ 


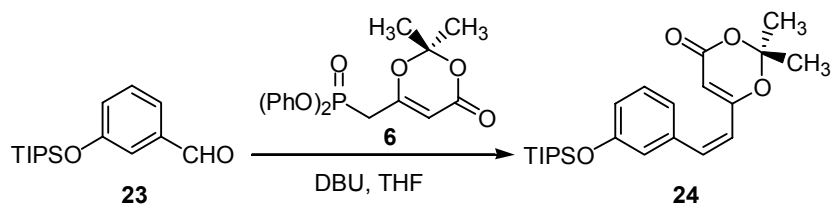

(Z)-Olefin 24. DBU (228 $\mu \mathrm{L}, 232 \mathrm{mg}, 1.52 \mathrm{mmol}, 2.0$ equiv) was added dropwise via syringe over $1 \mathrm{~min}$ to a stirring solution of phosphonate 6 (399 $\mathrm{mg}, 1.07 \mathrm{mmol}, 1.4$ equiv) in tetrahydrofuran $(5 \mathrm{~mL})$ at $23{ }^{\circ} \mathrm{C}$. After stirring for $20 \mathrm{~min}$ at $23{ }^{\circ} \mathrm{C}$, the reaction mixture was cooled to $0{ }^{\circ} \mathrm{C}$. A solution of aldehyde $23(212 \mathrm{mg}, 0.761 \mathrm{mmol}, 1$ equiv) in tetrahydrofuran (5.0 $\mathrm{mL}$ ) was added. The resulting mixture was allowed to stir at $0{ }^{\circ} \mathrm{C}$ for $30 \mathrm{~min}$. Saturated aqueous ammonium chloride solution $(30 \mathrm{~mL})$ was added, and the resulting biphasic mixture was concentrated in vacuo to remove tetrahydrofuran. The aqueous residue was extracted with ethyl acetate $(3 \times 30 \mathrm{~mL})$. The combined organic extracts were washed with brine $(2 \times 20 \mathrm{~mL})$. The washed organic solution was dried over anhydrous sodium sulfate. The dried solution was filtered and the filtrate was concentrated. The residue was purified by flash column chromatography (33\% ether-hexanes) to provide a 6.7:1 $(Z) /(E)$ mixture of olefin $24(277 \mathrm{mg}$, $90 \%$ ), which was used without further purification in the next reaction.

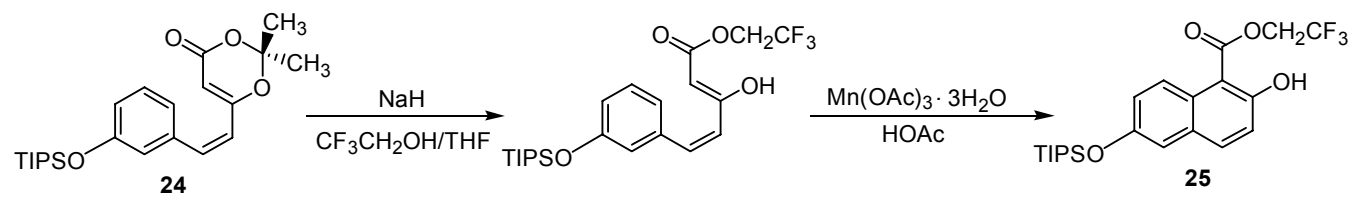

Trifluoroethyl 2-hydroxy-1-naphthoic acid ester 25. Sodium hydride (60\% dispersion in mineral oil, $53.7 \mathrm{mg}, 5.66 \mathrm{mmol}, 6.0$ equiv) was added to a stirring solution of trifluoroethanol (271 $\mu \mathrm{L}, 373 \mathrm{mg}, 3.73 \mathrm{mmol}, 10.0$ equiv) in tetrahydrofuran $(5 \mathrm{~mL})$ at $23{ }^{\circ} \mathrm{C}$. The reaction mixture was allowed to stir at $23{ }^{\circ} \mathrm{C}$ for $20 \mathrm{~min}$. A $(Z) /(E)$ mixture of olefin $24(150 \mathrm{mg}, 0.373$ mmol, 1 equiv, $\sim \mathrm{Z}: E 6: 1$, from the experiment above) in tetrahydrofuran $(5 \mathrm{~mL})$ was added via cannula. After stirring for $30 \mathrm{~min}$ at $23{ }^{\circ} \mathrm{C}$, the reaction mixture was concentrated in vacuo. The residue was taken up in acetic acid $(4 \mathrm{~mL})$. Manganese (III) acetate dihydrate (200 $\mathrm{mg}, 0.747$ mmol, 2.0 equiv) was added. After stirring for $1 \mathrm{~h}$ at $40{ }^{\circ} \mathrm{C}$, the reaction mixture was partitioned 
between water $(20 \mathrm{~mL})$ and ethyl acetate $(30 \mathrm{~mL})$. The aqueous layer was separated and extracted further with ethyl acetate $(2 \times 30 \mathrm{~mL})$. The organic extracts were combined and washed with saturated aqueous sodium bicarbonate solution $(2 \times 20 \mathrm{~mL})$. The washed organic solution was dried over anhydrous sodium sulfate. The dried solution was filtered and the filtrate was concentrated. The residue was purified by flash column chromatography $(10 \%$ etherhexanes) to give trifluoroethyl 2-hydroxy-1-naphthoic acid ester 25 as a clear colorless oil (111 mg, $61 \%$ over 3 steps).

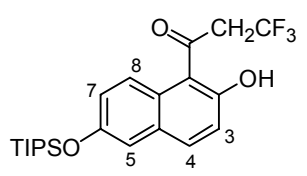

${ }^{1} \mathrm{H}$ NMR (500 MHz, $\left.\mathrm{CDCl}_{3}\right) \delta: \quad 11.62$ (s, 1H, C(2)-OH), 8.54 (d, J=9.5 Hz, 1H, C(8)-H), $7.80(\mathrm{~d}, \mathrm{~J}=9.5 \mathrm{~Hz}, 1 \mathrm{H}, \mathrm{C}(4)-\mathbf{H}), 7.23\left(\mathrm{dd}, \mathrm{J}_{1}=2.5 \mathrm{~Hz}, \mathrm{~J}_{2}=9.5\right.$ $\mathrm{Hz}, 1 \mathrm{H}, \mathrm{C}(7)-\mathbf{H}), 7.18$ (d, J=2.5 Hz, 1H, C(5)-H), 7.13 (d, $\mathrm{J}=9.0 \mathrm{~Hz}, 1 \mathrm{H}, \mathrm{C}(3)-\mathbf{H}), 4.87$ (q, J=8.5 Hz, 2H, C(1)$\left.\mathrm{C}(\mathrm{O}) \mathrm{OCH}_{2} \mathrm{CF}_{3}\right), 1.31\left(\mathrm{~m}, 3 \mathrm{H}, \mathrm{C}(6)-\mathrm{OSi}\left(\mathrm{CH}\left(\mathrm{CH}_{3}\right)_{2}\right)_{3}\right), 1.13$ $\left(\mathrm{d}, \mathrm{J}=7.5 \mathrm{~Hz}, 18 \mathrm{H}, \mathrm{C}(6)-\mathrm{OSi}\left(\mathrm{CH}\left(\mathrm{CH}_{3}\right)_{2}\right)_{3}\right)$

${ }^{13} \mathrm{C}$ NMR $\left(100 \mathrm{MHz}, \mathrm{CDCl}_{3}\right) \delta: \quad 170.6,163.9,152.4,136.9,130.1,126.4,126.2124 .1,123.0$ $(\mathrm{q}, \mathrm{J}=276 \mathrm{~Hz}), 119.4,116.6,103.4,61.0(\mathrm{q}, \mathrm{J}=37.2 \mathrm{~Hz})$, $17.9,12.7$

$\operatorname{HRMS}\left(\mathrm{CI}^{+}\right)$:

$m / z$ calcd for $\left(\mathrm{C}_{22} \mathrm{H}_{29} \mathrm{~F}_{3} \mathrm{O}_{4} \mathrm{Si}+\mathrm{NH}_{4}\right)^{+}$443.1866, found 443.1872

FTIR $\left(\mathrm{cm}^{-1}\right)$ : 3046, 2944, 2872, 1661, 1624, 1603, 1579, 1519, 1464, 1402 , 1352, 1326, 1283, 1208, 1254, 1208, 1166, 1134, $1067,988,971,882,843,828,797,758,686,651$ 